\title{
Research capacity of Australian and New Zealand emergency medicine departments, 2019 survey
}

Katie Walker ( $\square$ katie_walker01@yahoo.com.au )

Cabrini Hospital https://orcid.org/0000-0002-5313-5852

Shijie lan Tan

$\mathrm{Ng}$ Teng Fong General Hospital

Daniel Fatovich

Harry Perkins Institute of Medical Research

Gina Watkins

University of South Wales

Melanie Stephenson

Cabrini Hospital

Joseph Ting

Queensland University of Technology

Richard Whittome

Australasian College for Emergency Medicine

Wei Wang

Cabrini Institute

Jonathan Knott

Royal Melbourne Hospital

\section{Original Research}

Keywords: Emergency Medicine Research, Research personnel, Surveys and questionnaires, Health resources, Multicentre Trials

Posted Date: February 14th, 2020

DOI: https://doi.org/10.21203/rs.2.23588/v1

License: (c) (i) This work is licensed under a Creative Commons Attribution 4.0 International License. Read Full License

Version of Record: A version of this preprint was published at International Journal of Emergency Medicine on April 15th, 2020. See the published version at https://doi.org/10.1186/s12245-020-00275-z. 


\section{Abstract}

Background Large, multicentre studies are required in emergency medicine to advance clinical care and improve patient outcomes. The Australasian College for Emergency Medicine clinical trials network is available to researchers to assist with facilitating large, multicentre research. However, there is no current information about the research capacity of emergency departments (EDs) in Australia and New Zealand.

Methods All EDs accredited for emergency medicine training in Australia and New Zealand were eligible to participate. Research leads or ED directors were invited via email and telephone to complete a survey. Data were collected regarding the presence of a research lead, their research experience; available research resources including colleagues, funding, departmental paid research time; publications and research culture.

Results One hundred and twelve responses were received on behalf of $122(84 \%)$ sites (10 satellite plus main) from a possible 143 sites with all types of hospitals and regions represented. Research leads were identified at $66(59 \%)$ sites, 32 (29\%) had a director of emergency medicine research. A wide range of research was underway. Ninety-six sites $(66 \%)$ contributed data to multicentre projects. Twenty-one centres $(17 \%)$, were highly productive with multiple resources (skilled colleagues, funding, staffing), a positive research culture and high volume output. Sixty to seventy centres (50-58\%) had limited resources, experienced an unsupportive research culture and authored manuscripts infrequently. Paid time for research directors was associated with increased research outputs.

Discussion ACEM sites have capacity to undertake large multicentre studies with a varied network of sites and researchers. While some sites are well equipped for research, the majority of EDs had minimal research output.

\section{Background}

There are over 9 million emergency department attendances per annum in Australia $(1,2)$ and New Zealand(3). Whilst it is hoped that current clinical practice is best practice and based upon level 1 evidence, this is often not the case. As we evaluate treatment options, particularly for critically ill patients, we discover many current established therapies have limited efficacy $(4,5)$. Multicentre randomised studies have recently been published on core topics such as fluids in sepsis $(6,7)$, bronchiolitis management $(8,9)$, latrodectism $(10)$ and pneumothorax (11)recommending fundamental changes to patient management.

To undertake robust studies that properly answer questions about emergency therapies, large multicentre randomised clinical trials are needed. These enrol large numbers of patients, from diverse clinical settings, into well-designed and funded trials. Many patient conditions are identified sporadically in low numbers across hospital networks that vary in patient population and resourcing. Important trials increasingly require adequate funding and resources, skilled investigators and high-functioning clinical trial networks, capable of large-scale project design and delivery $(12,13)$.

Internationally there are a range of emergency medicine clinical trial networks participating in research. Some, such as the European Society for Emergency Medicine (EUSUM) research network facilitate trials, others groups undertake international multicentre studies (eg Asia, Australia and New Zealand Dyspnoea in Emergency Department (AANZDEM)). Some networks are well established, particularly in pediatrics (Pediatric Emergency Care Applied Research Network (PECARN); Paediatric Research in Emergency Departments International Collaborative (PREDICT)). Many jurisdictions or subspecialties of emergency medicine are forming new collaborations (eg Geriatric Emergency care Applied Research (GEAR) network). Networks can be national (New Zealand Emergency Medicine Network (NZEMN)) or international (PanAsian Trauma Outcomes Study (PATOS), clinical research network). In order for any of these networks to produce high-quality answers to research questions, individual sites must have the capacity to collect data.

The Australasian College for Emergency Medicine (ACEM) has had a clinical trials group delivering studies, which has recently reformed as a clinical trials network (ACEM CTN)(14). All 143 Australian emergency departments accredited to train emergency medicine residents (ACEM trainees) must support the research learning objectives in the ACEM training curriculum but the number of sites actively engaged in research has been unknown.

The goal of this study is to describe current research capacity at ACEM training accredited EDs by reporting current resources, funding, activity and research culture. We describe the skill sets and experience levels of emergency medicine research leads; sector and region personnel resources; available paid research time; funding; research outputs; ACEM research training places and research culture.

\section{Methods}


A survey was undertaken of all ACEM accredited EDs between February and April 2019, with invitations sent by email, with telephone followup for non-responders.

The survey was developed and piloted internally by the authors, being adapted from the Australian and New Zealand Intensive Care Society (ANZICS) survey(15), see Appendix 1. Survey instrument. Question structures were a combination of pick-lists, 5-point Likert scale items and free-text. Some questions were adaptive to responses to reduce the length of the survey and many sections were mandated. There was a completeness check prior to survey submission and participants were able to check their answers at any time prior to submission. Only one survey was allowed per site.

All Australian and New Zealand EDs that were accredited for ACEM training in November 2018 were eligible. Australian and New Zealand emergency medicine is largely funded by state and national governments, with universal health coverage for citizens and no out-of-pocket fees for patients. Emergency physicians are usually salaried employees of the government hospitals, with variable amounts of paid time available for non-patient facing work. In Australia, 7\% of patients attend private (non-governmental hospitals) and emergency physicians may be paid per hour or per patient as a salaried employee, without governmental support for non-patient facing work. Most university appointments for physicians are honorary/adjunct (unpaid).

EDs were identified from the ACEM accredited training sites database. ED Directors or their Directors of Emergency Medicine Research were invited to participate. The survey was advertised in the weekly ACEM bulletin email. Eligible emergency physicians were emailed a link to the voluntary survey and they (or delegate) were asked to complete it within two weeks. Phone calls were made to non-respondents. Informed consent was obtained; which included information about the research team, survey purpose, the likely time for completion of the survey and data privacy and protection. Data was collected from February 2019 to April 2019, until either a survey was complete, a site opted-out or several calls had been made without obtaining data. Missing data was followed up by phone. The survey was closed (invitees only) and was administered online (Redcap, version 8.8.1, Vanderbilt university, Tennessee, USA). The trained data collector (for phone responses) was IT, MS, or GW.

Data collected from the ACEM database were site demographics, locations, training accreditation categories and role delineations. Data were collected about emergency physicians and ACEM trainees were research titles, skills, qualifications and experience levels and the amount of paid research time allocated. Departmental research interests, publication volume, funding amounts and sources for the preceding five years was obtained. Publications were defined as: "published in a peer-reviewed Medline-listed research publication including at least one ED member as an author". Grants were defined as: "funded grants (The ED must be a major partner in the grant application eg principal investigator or major clinical health partner)." Non-emergency physician research staff information was also sought (types of personnel and paid hours available). Qualitative information regarding site research culture was obtained.

All data from complete surveys were included in the analysis. Incomplete/early-termination survey data was also included in the analysis. Descriptive data was summarised using number (\%) or median (interquartile range). Likert scores were grouped into negative (very unimportant, not important, neutral) and positive (somewhat important, very important) and reported as percentages. Free text was analysed by two reviewers (MS, KW) using an inductive approach and open coding for themes and content with examples presented.

\section{Results}

\section{Participant flow}

Study flow proceeded as described in Figure 1, with 112 survey responses (with 10 people providing combined responses about a main and satellite site: data from 122 physical sites total) from an initial potential 145 sites.

\section{Participant characteristics}

The site characteristics of survey participants are available in Table 1.

Table 1. Demographics of sites and participating sites 


\begin{tabular}{|c|c|c|}
\hline Regions & Total sites number & Responses number (\%) \\
\hline Australian Capital Territory & 2 & $1(50 \%)$ \\
\hline New South Wales & 37 & $26(70 \%)$ \\
\hline New Zealand & 18 & $13(72 \%)$ \\
\hline Northern Territory & 2 & $2(100 \%)$ \\
\hline Queensland & 26 & $24(92 \%)$ \\
\hline South Australia & 7 & $6(86 \%)$ \\
\hline Tasmania & 2 & $2(100 \%)$ \\
\hline Victoria & 26 & $25(96 \%)$ \\
\hline Western Australia & 13 & $13(100 \%)$ \\
\hline \multicolumn{3}{|c|}{ Aust. Inst. of Health and Welfare, hospital classifications } \\
\hline (New Zealand) & 18 & $13(72 \%)$ \\
\hline Private & 13 & $12(92 \%)$ \\
\hline Large regional & 21 & $20(95 \%)$ \\
\hline Medium regional & 2 & $2(100 \%)$ \\
\hline Small/Medium regional & 6 & $5(83 \%)$ \\
\hline Major & 30 & $26(87 \%)$ \\
\hline Large metropolitan & 27 & $24(89 \%)$ \\
\hline Medium metropolitan & 10 & $8(80 \%)$ \\
\hline Specialist children's & 6 & $2(33 \%)$ \\
\hline \multicolumn{3}{|l|}{ Age of patients } \\
\hline Adults and pediatric & 121 & $105(87 \%)$ \\
\hline Adults & 5 & $5(100 \%)$ \\
\hline Pediatric & 7 & $2(29 \%)$ \\
\hline \multicolumn{3}{|l|}{ ACEM site classifications } \\
\hline Major referral & 38 & $31(82 \%)$ \\
\hline Rural/regional base & 45 & $38(84 \%)$ \\
\hline Urban District & 50 & $43(86 \%)$ \\
\hline \multicolumn{3}{|c|}{ Hospital education accreditation time for each ACEM trainee/resident } \\
\hline 6 months & 37 & $32(86 \%)$ \\
\hline 12 months & 35 & $29(83 \%)$ \\
\hline 18 months & 19 & $12(63 \%)$ \\
\hline 24 months & 42 & $39(93 \%)$ \\
\hline Total & 133 & 112 \\
\hline
\end{tabular}

Overall, there was an $84 \%$ response rate; which was evenly spread across types of hospitals and regions. There was a lower response rate from specialist children's hospitals, hospitals accredited for 18 months of training and a few states.

Sixty-six sites (59\%) had appointed a head of research and 32 (29\%) held the title of Director of Emergency Medicine Research or similar. All except 2 heads of research were Fellows of the Australasian College for Emergency Medicine (the others both hold a PhD). Amongst the heads of research, there were 12 Associate Professors and 8 Professors. We didn't distinguish between adjunct/honorary and full university appointments. Forty-nine (74\%) heads of research have a university affiliation, 28 (42\%) have been a site chief investigator for a multisite project, 18 (27\%) have been a principal investigator on a National Health and Medical Research Council grant. Forty-two university research degrees were awarded to 33 research heads (12 Doctoral degrees, 5 Doctors of Medicine, 13 Masters degrees and 12 other/unknown Higher Research Degrees). The levels of experience of heads of research are shown in Figure 2. 
Types of research

Departmental research interests varied widely, reflecting the breadth of emergency medicine practice environments and populations (Figure 3). Ninety-six EDs contributed to multicentre research during the last 5 years. Fifty-two EDs contributed to ACEM CTN projects, mainly collecting data for the ARISE sepsis fluids observational study $(n=52)(16)$ and the spontaneous pneumothorax study $(n=19)(11)$.

\section{Publications}

Overall site contributions to publications were 3336 papers in the last five years from the 112 sites. This figure was calculated by summing the number of papers per site but there will have been overlap for multi-site investigations such that the true total number will be lower. Publication volume varied markedly. The 32 sites with a Director of Emergency Medicine Research (or equivalent) appointed published a median of 26.5 papers ( $\mathrm{n}=1814$; IQR 8,67; range 0,302). The 33 sites with a head of research (excluding those with a Director of Emergency Medicine Research) published a median of 6 papers ( $n=1237$; IQR 1.5,13; range 0,502) compared to median of 0 from the 47 sites without a head of research $(n=193$; IQR 0,3; range 0,92). There were 34 sites that didn't author a publication in the last 5 years.

\section{Funding}

The total funding achieved over the last 5 years was approximately $\$ 71$ million AUD (Figure 4). Of this, \$43.1 million was obtained from a national/federal government medical research council and approximately \$3-7 million each was obtained from philanthropy, hospital foundations, other foundations, state governments, other federal government grants and from block industry grants.

Major metropolitan and specialist children's hospitals were the most successful in obtaining grants, small to medium hospitals (metropolitan or regional) the least successful. Four regions performed better than others in obtaining funds per head of population (NZ, QLD, VIC, WA) with the other regions obtaining significantly less. The distribution of funding per site was highly skewed.

The median per site value was $\$ 0$ (IQR \$0,\$107.5K; range $\$ 0, \$ 21$ million). Sixty-six of 112 sites didn't achieve any funding and a further 20 achieved less than $\$ 150 \mathrm{~K}$. Seven sites obtained over $\$ 500 \mathrm{~K}, 5$ over $\$ 1$ million, and the remaining 12 achieved $\geq \$ 1.5$ million in grant funding. Sites with a Director of Emergency Medicine Research obtained a median of $\$ 105,000$ in grants (IQR 5.25,810K), sites without a Director of Emergency Medicine Research obtained a median of $\$ 0$ in grants (head of research IQR 0,37K; no research lead IQR 0,3K).

\section{Staffing}

Eighty-seven sites had emergency physicians conducting research (median 2, IQR 1,4). The median Fulltime Equivalent paid emergency physician research hours was 0.1 (IQR 0,0.25) or 3.8 hours per week. Post-doctoral researchers and other paid researchers worked at 61 sites (median 0, IQR 0,0.5). Most sites had limited resources in terms of personnel 
and were reliant on volunteer labour (emergency physicians, other ED staff and 173 medical students). Two sites paid multiple ACEM trainees to conduct research and eight others employed 1-2 trainees.

At the 66 sites with heads of research, the 32 Directors of Emergency Medicine Research were paid a median of 10 research hours per week (IQR 5,20; range 0,40). When the head of research didn't have the Director of Emergency Medicine Research title (33 people) the median paid research time per week was 0 hours per week (IQR 0,5.5; range $0,10)$.

\section{$\underline{\text { Culture }}$}

Regarding questions about research culture at the EDs, 55\% (62/112) thought that emergency medicine research was important to their organisations. Most thought that emergency medicine research was important to their EDs (72\%, 81/112). Research leads felt less supported by their fellow emergency physicians with 54\% (60/112) giving positive responses and by other ED staff (eg nursing co-workers) with 46\% (51/112) positive responses. Most felt unsupported when conducting clinical research with a 70\% (78/112) negative response rate. Sectors and regions giving more optimistic responses across all questions included specialist children's, medium regional and New Zealand hospitals. Those struggling included private and medium metropolitan and South Australian hospitals. Free-text responses from 63 respondents identified 3 major and 3 minor themes. Theme saturation was achieved.

Major themes

1. Support

Some felt well supported: "We have been well supported by the hospital", "We have good support from colleagues for studies and it is a warm and welcoming environment". Others were supported in theory without practical support: "Organisation expresses commitment but this is not resourced or matched by reality". Another group felt completely unsupported: "Deprioritised against service provision", "no time, support or encouragement locally".

\section{Importance of research at the site}

There were variable thoughts amongst colleagues regarding the importance of research: "It is important for emergency physicians in this ED to be exposed to research projects", "some (emergency physicians) do not feel clinical research has a role (for them), I have been asked 'what's in it for me?", "we have very active nursing research".

\section{Research infrastructure as a barrier or enabler}

"Support from the Emergency Medicine Foundation research support network has been invaluable", "poor infrastructure and support processes create active barriers". When recruiting patients into clinical trials, some had colleagues who actively supported projects, others declined to recruit patients. 
Minor themes (practical research training, mentoring and advocacy for research)

Some felt there had been a decline in practical clinical research training since the change to ACEM fellowship training research requirements (allowing course completion instead of manuscript publication or presentation) "we are not developing clinical scientists", "...created a mindset that there was no benefit to do research" others advocated for mentoring and senior advice for those expressing an interest in clinical research as a career: "...how to set up a research culture....how to actually go about research..". Finally, there was a need identified for advocacy for the importance of research to enable prioritisation of and resource allocation into research, particularly as many felt there were no resources currently available to them "without resources/funding research isn't possible".

\section{Discussion}

This study found an emergency medicine research landscape that is varied. There is research capacity in all types of hospitals and regions with the resources to undertake multi-centre studies. Multiple researchers staff 21 centres (17\%), which have excellent infrastructure support, a supportive culture, multiple grants and publications. Sixty to seventy sites rely on volunteers; in-kind donations for infrastructure; experience a lack of funds and have an unsupportive research culture.

This is the first description of the emergency medicine research landscape in Australia and New Zealand. Our findings were similar to the ANZICS research capacity study(15). Emergency medicine has a higher rate of appointment of a head of research (66\% ED, 34\% ANZICS), and these researchers have similar levels of skills and experience. A similar proportion of sites were contributing to multicentre studies (86\% ED, $80 \%$ ANZICS). Emergency medicine had less help from research assistants or coordinators (28\% ED, 36\% ANZICS).

There is an association between engagement of individuals and organisations with research and improved healthcare performance(17) and patient survival $(18,19)$, although this hasn't been evaluated in emergency medicine. Having and paying a Director of Emergency Medicine Research to organise the department's research, was significantly associated with increased departmental research grants and publications. An ED could potentially improve its research contribution by appointing a Director of Emergency Medicine Research and allocating them paid non-clinical support time.

To put emergency medicine research funding into context compared to potential Australian and New Zealand national funding sources; in Australia \$8.8 billion was available to health via the National Health and Medical Research Council (NHMRC) and Medical Research and Futures Fund (MRFF)(20) over the last 5 years and the Australian EDs achieved $\$ 35.1$ million or $0.4 \%$ of these funds (whilst managing $10 \%$ of clinical encounters). Similarly, Australian philanthropic funds were approximately $\$ 55$ billion(21), whilst philanthropic funding achieved by Australian EDs was $\$ 3.4$ million $(0.006 \%)$.

It was heartening to note that many sites expressed interest or were conducting research despite currently feeling that they were being hampered by a lack of resources or governance issues. This group of sites represents untapped potential that with a little support should be able to deliver patient trial data representing their clinical populations.

Departments with a nominated research lead (without funding or allocated research time) undertook more research than those without a nominated lead. An initial step to facilitate multi-site research (without increasing overhead costs) might be to identify a research lead for each site, so that researchers initiating multi-site projects are able to invite diverse sites to participate in site data collection.

\section{Limitations}

Retrospective survey methods may introduce non-response, acquiescence, demand characteristic and desirability bias. Pediatric EDs were under-represented. The study focused mainly on emergency medicine research and may (or may not) have under-reported emergency medicine research undertaken by other disciplines such as nursing and allied health groups. Respondents usually only had access to information about themselves and overlapping information would lead to overestimation of grants and publications. There was no attempt made to verify responses for correctness using external sources. Research culture responses may be biased in either direction as researchers and directors provided data. Some regions have centralised research infrastructure and resources, uncaptured by this evaluation. 
Site circumstances change over time and misrepresentations of research lead classifications may have occurred. Many sites recently appointed Directors of Emergency Medicine Research to assist with training accreditation. Some questions relate to the preceding 5 years of research outputs and so may underestimate the impact of appointing and paying a Director of Emergency Medicine Research.

\section{Conclusions}

ACEM sites have capacity to undertake large multicentre studies with a varied network of sites and researchers. While some sites are well equipped for research, the majority of EDs had minimal research output.

\section{Declarations}

\section{Ethics approval and consent to participate}

Ethics approval was obtained from Cabrini Human Research Ethics Committee (01-29-10-18). Informed consent was obtained from all participants.

\section{Consent for publication}

Not applicable

\section{Availability of data and materials}

The datasets generated and analysed during the current study are not publically available to protect participant privacy.

\section{Competing Interests}

Many of the authors are members of the ACEM Clinical Trials Network.

\section{Funding}

No grants were obtained to fund this study. In-kind infrastructure and research support: Cabrini Institute, Cabrini Technology, Royal Melbourne Hospital. The Australasian College for Emergency Medicine contributed to journal publication fees. ACEM had no role in the design of the study, collection, analysis and interpretation of data or in writing the manuscript.

\section{Authors' contributions}

Resourcing KW, JK; Study design KW, JK, DF; Ethics KW, IT; Data instrument KW, JK, IT; Data collection IT, MS, GW, KW, RW; Data analysis KW, IT, MS, WW; Manuscript draft KW, JK; Manuscript revision all authors. All authors read and approved the final manuscript.

\section{Acknowledgements}

Maggie Bock, RMH - survey instrument; Michael Stokes - Redcap access; ACEM - accredited site data; Stephen MacDonald, Peter Jones, Gerben Keijzers, Tegwen Howell.

\section{References}

1. Australian Bureau of Statistics. 4390.0 - Private Hospitals, Australia, 2014-15, Private Acute and Psychiatric Hospitals - Summary Tables [Internet]. Canberra ACT: Australian Bureau of Statistics; 2016. Available from:

https://www.abs.gov.au/AUSSTATS/abs@.nsf/DetailsPage/4390.02014-15?OpenDocument. Accessed: 2019 Jul 15. 
2. Australian Institute of Health and Welfare. Emergency department care 2016-17: Australian hospital statistics [Internet]. 2017. Canberra ACT: Australian Institute of Health and Welfare; 2017. Available from: https://www.aihw.gov.au/reports/hospitals/ahs-2016-17emergency-department-care/data. Accessed: 2019 Jul 15.

3. Ministry of Health, Manatu Hauora. Emergency department use 2014/15 [Internet]. Wellington, New Zealand: Ministry of Health, Manatu Hauora; 2016. Available from: https://www.health.govt.nz/publication/emergency-department-use-2014-15. Accessed: 2019 Jul 15.

4. Baker B, Kessler K, Kaiser B, Waller R, Ingle M, Brambilla S, et al. Non-traumatic musculoskeletal pain in Western Australian hospital emergency departments: A clinical audit of the prevalence, management practices and evidence-to-practice gaps. Emergency medicine Australasia : EMA. 2019.

5. Schuh S, Babl FE, Dalziel SR, Freedman SB, Macias CG, Stephens D, et al. Practice Variation in Acute Bronchiolitis: A Pediatric Emergency Research Networks Study. Pediatrics. 2017;140(6).

6. Macdonald SPJ, Keijzers G, Taylor DM, Kinnear F, Arendts G, Fatovich DM, et al. Restricted fluid resuscitation in suspected sepsis associated hypotension (REFRESH): a pilot randomised controlled trial. Intensive Care Med. 2018;44(12):2070-8.

7. Investigators A, Group ACT, Peake SL, Delaney A, Bailey M, Bellomo R, et al. Goal-directed resuscitation for patients with early septic shock. N Engl J Med. 2014;371(16):1496-506.

8. Franklin D, Babl FE, Schlapbach LJ, Oakley E, Craig S, Neutze J, et al. A Randomized Trial of High-Flow Oxygen Therapy in Infants with Bronchiolitis. New England Journal of Medicine. 2018;378(12):1121-31.

9. O'Brien S, Borland ML, Cotterell E, Armstrong D, Babl F, Bauert P, et al. Australasian bronchiolitis guideline. Journal of Paediatrics and Child Health. 2019;55(1):42-53.

10. Isbister GK, Page CB, Buckley NA, Fatovich DM, Pascu O, MacDonald SPJ, et al. Randomized Controlled Trial of Intravenous Antivenom Versus Placebo for Latrodectism: The Second Redback Antivenom Evaluation (RAVE-II) Study. Annals of emergency medicine. 2014;64(6):620-8.e2.

11. Brown SGA, Ball EL, Perrin K, Asha SE, Braithwaite I, Egerton-Warburton D, et al. Conservative versus Interventional Treatment for Spontaneous Pneumothorax. New England Journal of Medicine. 2020;382(5):405-15.

12. Brown SGA, Isbister GK. Clinical research is a priority for emergency medicine but how do we make it happen, and do it well? Emergency Medicine Australasia. 2014;26(1):14-8.

13. KPMG. NIHR Clinical Research network: Impact and Value Assessment [Internet]. United Kingdom: KPMG; 2016. Available from: https://www.nihr.ac.uk/life-sciences-

industry/documents/NIHR\%20CRN\%20Impact\%20and\%20Value\%20FINAL\%20REPORT_vSTC_160908_FOR\%20EXTERNAL\%20USE.pdf. Accessed: 2019 Jul 15

14. Australasian College for Emergency Medicine. ACEM Clinical Trials Network [Internet]. Melbourne, Australia: Richard Whittome and ACEM CTN executive; 2018. Available from: https://acem.org.au/Content-Sources/Members/Research-at-ACEM/ACEM-Clinical-TrialsNetwork. Accessed 2019 Jul 15.

15. Goulding KR, Peyton PJ, Story DA, Parker A, Leslie K. Survey of research capability at Australian and New Zealand college of anaesthetists accredited training sites.(Report). Anaesthesia and intensive care. 2017;45(2):196.

16. Keijzers G, Macdonald SPJ, Udy AA, Arendts G, Bailey M, Bellomo R, et al. The Australasian Resuscitation In Sepsis Evaluation: FLUid or vasopressors In Emergency Department Sepsis, a multicentre observational study (ARISE FLUIDS observational study): Rationale, methods and analysis plan. Emergency Medicine Australasia. 2019;31(1):90-6.

17. Boaz A, Hanney S, Jones T, Soper B. Does the engagement of clinicians and organisations in research improve healthcare performance: a three-stage review. BMJ Open. 2015;5(12):e009415.

18. Downing A, Morris EJ, Corrigan N, Sebag-Montefiore D, Finan PJ, Thomas JD, et al. High hospital research participation and improved colorectal cancer survival outcomes: a population-based study. Gut. 2017;66(1):89-96.

19. Rochon J, du Bois A, Lange T. Mediation analysis of the relationship between institutional research activity and patient survival. BMC Med Res Methodol. 2014;14:9.

20. Parliament of Australia. Science and Research Funding, Budget review 2015-16 [Internet]. Canberra ACT: Parliamentary Library; 2015. Available from:

https://www.aph.gov.au/About_Parliament/Parliamentary_Departments/Parliamentary_Library/pubs/rp/BudgetReview201516/Science. Accessed 2019 Jul 15.

21. Philanthropy Australia. Giving Australia, Report on Philanthropy and Philanthropists [Internet]. Melbourne Australia: Australian Centre for Philanthropy and Nonprofit studies, QUT; 2017. Available from: https://www.philanthropy.org.au/giving-australia. Accessed 2019 Jul 15. 
Figures

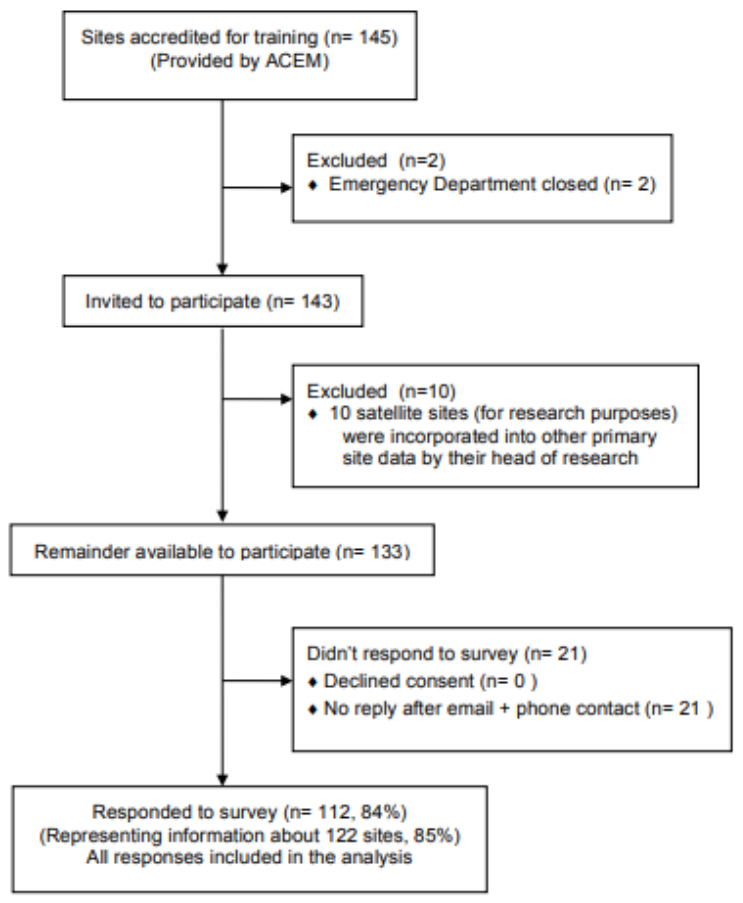

Figure 1

Consort Flow Diagram for ACEM research capacity study 2019

\section{Levels of experience of heads of research}

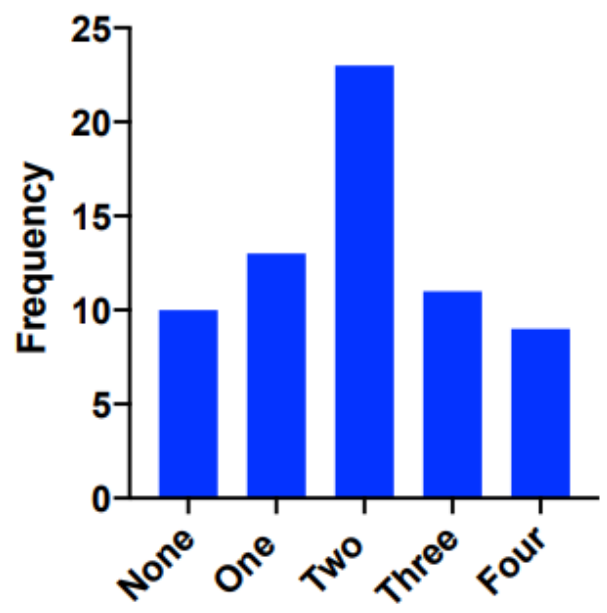

Total number of sites $n=112$

Total heads of research $n=66$

Experience attributes:

- University research qualification

- University affiliation

- Local chief investigator for multisite study

- Principal investigator for NHMRC or equivalent grant

\section{Number of attributes per head of research}

Figure 2

Levels of experience of heads of research 


\begin{tabular}{|c|c|}
\hline $\begin{array}{l}\text { Types of evaluations } \\
\text { Disease Specific }\end{array}$ & \\
\hline Anaesthetics & Psychiatry \\
\hline Cardiology & Respiratory \\
\hline Cognition & Resuscitation \\
\hline Infectious Disease & Sepsis \\
\hline Neurology & Toxicology \\
\hline Neurosurgery & Toxinology \\
\hline Utility of investigations & \\
\hline Pathology & Radiology \\
\hline Clinical Decision instruments & \\
\hline Emerging/Alternate therapies & \\
\hline Immunotherapy & Acupuncture \\
\hline Others & \\
\hline Emergency processes & Novel technology \\
\hline Emergency systems & Pain management \\
\hline Health economics & Patient Satisfaction \\
\hline Information technology & Public health \\
\hline Metadata use & Staff welfare/safety \\
\hline External environments & \\
\hline $\begin{array}{l}\text { Battlefield medicine } \\
\text { Age-specific }\end{array}$ & Pre-hospital \\
\hline Geriatrics & Paediatrics \\
\hline
\end{tabular}

Figure 3

Types of research undertaken at ACEM sites

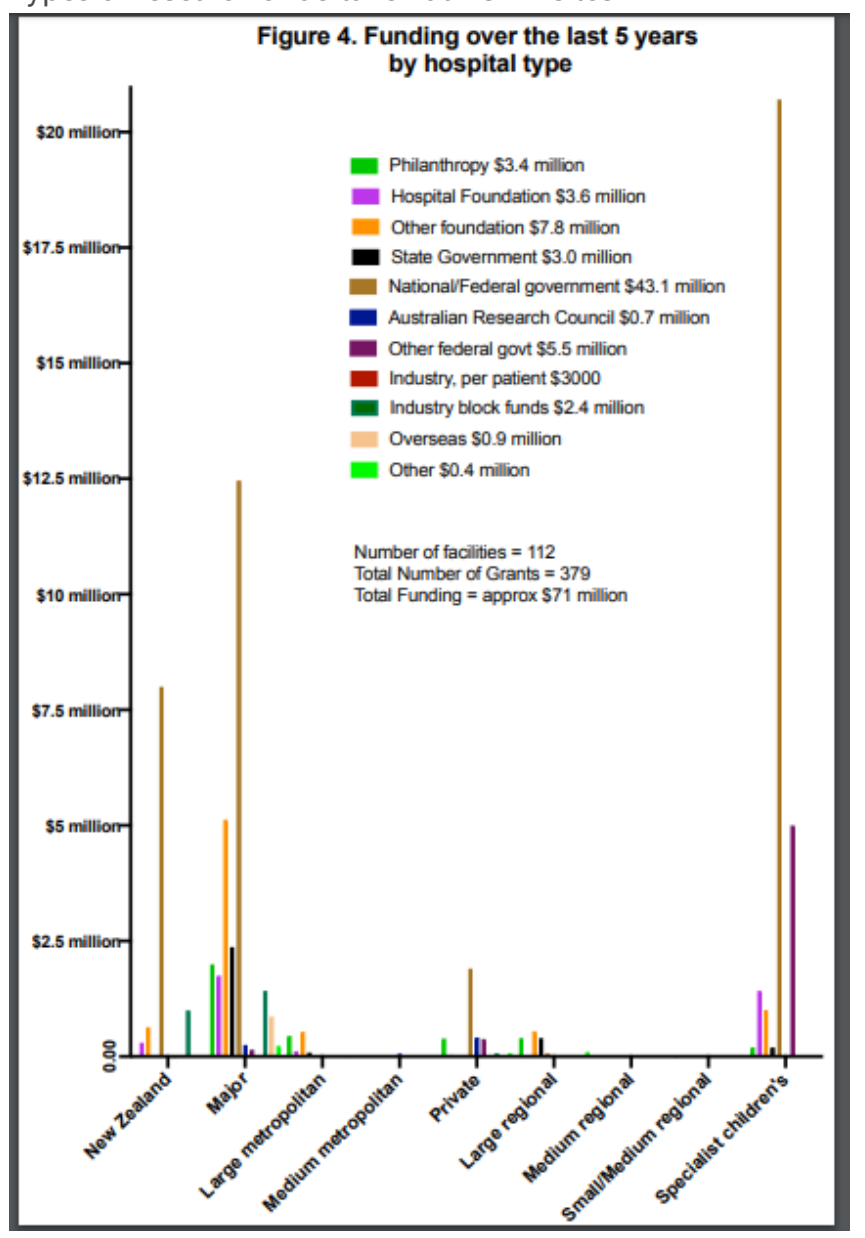

Figure 4 
Funding over the last 5 years by hospital type

\section{Supplementary Files}

This is a list of supplementary files associated with this preprint. Click to download.

- Appendix1.Onlinesurvey.docx 